\title{
Philosophiques
}

\section{L'hystérie : ne plus vouloir pouvoir, ne plus pouvoir vouloir}

\section{Frédérique De Vignemont}

Volume 33, numéro 1, printemps 2006

Philosophie et psychopathologie

URI : https://id.erudit.org/iderudit/012953ar

DOI : https://doi.org/10.7202/012953ar

Aller au sommaire du numéro

\section{Éditeur(s)}

Société de philosophie du Québec

\section{ISSN}

0316-2923 (imprimé)

1492-1391 (numérique)

Découvrir la revue

\section{Citer cet article}

De Vignemont, F. (2006). L'hystérie : ne plus vouloir pouvoir, ne plus pouvoir vouloir. Philosophiques, 33(1), 197-215. https://doi.org/10.7202/012953ar

\section{Résumé de l'article}

L'hystérie se définit comme un déficit fonctionnel sans cause organique. Par exemple, certains patients sont incapables de se mouvoir volontairement, comme s'ils étaient véritablement paralysés, sans que l'on puisse fournir une explication physiologique. À l'inverse, les patients souffrant d'anosognosie sont véritablement paralysés, mais affirment pouvoir bouger. Ces pathologies résultent toutes deux d'un trouble de la conscience de la capacité à agir : les uns croient qu'ils ne peuvent pas agir alors qu'ils le pourraient et les autres croient pouvoir agir alors qu'ils en sont incapables. Mais de quoi dépend cette forme spécifique de conscience ? En comparant ces deux syndromes miroirs, je chercherai à révéler les processus mis en jeu par la conscience de la capacité à agir et à jeter un jour nouveau sur l'hystérie. S'agit-il d'un trouble de la volonté, et si oui, à quel niveau se produit-il ? J'analyserai ainsi les relations respectives entre la conscience de la capacité d'agir et la conscience d'agir, et montrerai le rôle joué par l'image corporelle.
Tous droits réservés (C) Société de philosophie du Québec, 2006
Ce document est protégé par la loi sur le droit d'auteur. L'utilisation des services d'Érudit (y compris la reproduction) est assujettie à sa politique d'utilisation que vous pouvez consulter en ligne.

https://apropos.erudit.org/fr/usagers/politique-dutilisation/ 


\title{
L'hystérie : ne plus vouloir pouvoir, ne plus pouvoir vouloir
}

\author{
FRÉDÉRIQUE DE VIGNEMONT \\ Institut des sciences cognitives, CNRS Lyon \\ fvignemont@isc.cnrs.fr
}

\begin{abstract}
RÉSUMÉ. - L'hystérie se définit comme un déficit fonctionnel sans cause organique. Par exemple, certains patients sont incapables de se mouvoir volontairement, comme s'ils étaient véritablement paralysés, sans que l'on puisse fournir une explication physiologique. À l'inverse, les patients souffrant d'anosognosie sont véritablement paralysés, mais affirment pouvoir bouger. Ces pathologies résultent toutes deux d'un trouble de la conscience de la capacité à agir : les uns croient qu'ils ne peuvent pas agir alors qu'ils le pourraient et les autres croient pouvoir agir alors qu'ils en sont incapables. Mais de quoi dépend cette forme spécifique de conscience ? En comparant ces deux syndromes miroirs, je chercherai à révéler les processus mis en jeu par la conscience de la capacité à agir et à jeter un jour nouveau sur l'hystérie. S'agit-il d'un trouble de la volonté, et si oui, à quel niveau se produit-il ? J'analyserai ainsi les relations respectives entre la conscience de la capacité d'agir et la conscience d'agir, et montrerai le rôle joué par l'image corporelle.
\end{abstract}

\section{Introduction}

Dans une perspective de naturalisation de la conscience, et plus particulièrement de la conscience d'agir, il apparaît nécessaire de s'appuyer sur les données empiriques issues des sciences cognitives pour redéfinir les problèmes philosophiques et rechercher des réponses nouvelles en dehors de leur cadre conceptuel d'origine. La psychopathologie joue alors un rôle majeur pour l'étude du fonctionnement normal de l'esprit. Il faut, non pas réduire les troubles psychiatriques à de simples affabulations sans fondement, mais au contraire comprendre leur genèse causale et les interpréter comme résultant d'un dysfonctionnement cognitif. Une réflexion sur l'origine de certains phénomènes psychiatriques peut ainsi apporter un éclairage nouveau sur certaines questions philosophiques. Cet article s'inscrit donc dans ce que nous pouvons désormais qualifier de "philosophie de la psychopathologie » (Graham et Stephens, 1994).

L'étude de la schizophrénie illustre de manière frappante l'intérêt de la psychopathologie pour la philosophie de l'esprit. Elle a suscité ces dix dernières années une vaste littérature, tant scientifique que philosophique, et permis de formuler de nouvelles hypothèses sur la conscience de soi. Frith (1992) soutient ainsi que les symptômes positifs de la schizophrénie, tels que le délire de contrôle et les hallucinations verbales, doivent être interprétés comme des troubles de la conscience de soi comme agent (ou du sens de l'agentivité) : les patients ne se reconnaissent plus à l'origine de leurs propres 
actions motrices ou verbales et préfèrent les attribuer à autrui ; ils se sentent alors télécommandés ou entendent une voix leur parler. À partir de l'analyse des processus de l'action, Frith a suggéré l'existence d'un système de contrôle interne sous-jacent au sens de l'agentivité, reposant sur l'anticipation des conséquences de nos actes et sur leur comparaison avec leurs effets actuels. L'étude de la schizophrénie et le modèle de Frith ont permis de distinguer différents niveaux de représentation de l'action : le contrôle de l'action, la conscience de l'action et le sens de l'agentivité (Proust, 2000 ; de Vignemont et Fourneret, 2003). Le contrôle s'effectue de façon automatique, subconsciente et rapide. Il repose sur des représentations motrices très détaillées de la position corporelle et de l'environnement. Au contraire, la conscience de l'action est limitée et peu précise. L'agent n'a principalement accès qu'à son intention préalable (j'ai l'intention de saisir ce verre), mais ignore les paramètres spécifiques de ses mouvements. Le sens de l'agentivité se situe à l'intersection de ces deux plans. Il ne se confond pas intégralement avec le contrôle de l'action, car à la différence de ce dernier qui se situe dans un référentiel solipsiste, l'attribution de l'action vise à distinguer soi d'autrui. Il diffère aussi de la conscience de l'action. D'une part en effet, je n'ai pas besoin d'avoir conscience de l'action pour pouvoir la reconnaître comme mienne (comme dans la plupart des actions automatiques), et d'autre part, je peux avoir conscience de l'action sans la reconnaître pour autant comme mienne (comme dans le cas du délire de contrôle).

Je n'approfondirai pas ici la question de la schizophrénie ni même celle du sens de l'agentivité. Je vais plutôt tenter d'explorer une pathologie tombée un peu en désuétude, mais qui a connu un regain d'intérêt ces dernières années : l'hystérie de conversion. En quelques mots, l'hystérie se définit comme un déficit fonctionnel sans cause organique. Elle est diagnostiquée dans 1 à $3 \%$ des cas dans la pratique neurologique (Marsden, 1986). Je mettrai plus particulièrement l'accent sur la paralysie hystérique : les patients disent ne pas pouvoir bouger, comme s'ils étaient véritablement paralysés, sans qu'il y ait pour autant de dommage moteur ni périphérique ni sur le plan neurologique. Ils sont pourtant incapables de réaliser des actions volontaires. Seuls leurs réflexes et certains mouvements automatiques sont préservés. L'hystérie apparait alors comme un trouble de l'action. Il ne s'agit pas ici d'un déficit du sens de l'agentivité ni même d'un désordre de la conscience de l'action : de facto les patients ne peuvent plus agir. Il paraît donc nécessaire de postuler un niveau supplémentaire de représentation de l'action qui serait perturbé chez les patients hystériques, celui de la conscience de la capacité d'agir.

\section{Les troubles de la conscience de nos capacités}

Quand je vous demande si vous pouvez lever votre bras droit, vous êtes en position de répondre par l'affirmative sans même avoir à effectuer le mouvement demandé. Vous savez en effet que vous n'êtes pas paralysé et que vous êtes libre de vous mouvoir. Il vous paraît naturel de connaître à tout instant 
vos capacités, ainsi que vos limites. En ce sens, la conscience de l'action n'est pas la conscience d'une action particulière, mais celle de la capacité d'agir. Toutefois, elle n'est pas sans limite, comme le montrent certains phénomènes neuropsychologiques et psychopathologiques. Plus particulièrement, l'étude de la paralysie hystérique permet de mieux comprendre les processus sousjacents à la connaissance de nos capacités.

\subsection{Le syndrome de conversion hystérique}

« Dans l'hystérie de conversion, le corps possède le pouvoir de représenter les désordres les plus aléatoires, sans encourir de danger » (John Ferriar, 1794, p. 12).

Un siècle avant Freud, Ferriar s'intéressait d'ores et déjà à l'hystérie de conversion, sans pour autant chercher à en comprendre l'origine. Ce n'est qu'au $\mathrm{XIX}^{\mathrm{e}}$ siècle que différentes explications ont été proposées, tant sur le plan cérébral et psychologique que gynécologique ou érotologique ${ }^{1}$. D'abord conçue comme le résultat d'une excitabilité accrue du système nerveux périphérique, l'hystérie a été ensuite considérée comme le reflet d'une lésion "fonctionnelle » du cortex moteur (Charcot, 1889) ou d'un déficit du contrôle central sur les processus de bas niveau. À ces explications neurologiques se sont opposées celles plus psychologiques qui mettent l'accent sur le rôle déclencheur joué par les émotions (Carter, 1853) ou par le résidu inconscient d'une idée fixe (Janet, 1907). Freud a alors constitué la synthèse de ces différents courants de pensée, en développant la notion de conversion d'un trauma psychologique probablement d'origine sexuelle en symptômes physiques : "Dans l'hystérie, l'idée incompatible est rendue inoffensive par la somatisation de la somme des excitations " (Freud, 1894, p. 49).

De nos jours, trois principes gouvernent la notion d'hystérie de conversion :

1. Les symptômes sont similaires à ceux rencontrés dans une maladie organique, mais ne découlent d'aucune origine biologique connue. Ils peuvent affecter la perception, la mémoire, la motricité, la douleur...

2. Les patients ont l'impression de n'avoir aucun contrôle volontaire sur leurs symptômes et les ressentent comme réels. Cependant, ils n'ont pas une réaction appropriée vis-à-vis de la gravité de leur état et éprouvent ce que Janet a qualifié de "belle indifférence ».

3. Comme les symptômes n'ont pas d'explication organique, ils doivent être provoqués par des facteurs psychologiques. Ainsi, ils résulteraient de la " conversion » de perturbations psychologiques en symptômes physiques.

Le diagnostic de l'hystérie présente de nombreuses difficultés, et ce d'autant plus que les symptômes sont très variés et que l'étiologie demeure inconnue,

1. Pour un historique plus complet, voir Mace (2001). 
comme Ferriar l'avait déjà remarqué il y a plus de deux cents ans. La classification même de l'hystérie est difficile, certains préférant la ranger dans les désordres somatoformes (DSM-IV), tandis que d'autres la définissent comme un désordre dissociatif. Se pose en outre la question de savoir si l'hystérie existe réellement (Miller, 2001). En premier lieu, il est possible d'imaginer que le fait de ne pas avoir trouvé de causes physiologiques n'implique pas qu'elles n'existent pas. Certains patients étiquetés comme hystériques se sont ainsi révélés par la suite souffrir d'une maladie physique (Slater et Glithero, 1965). De plus, et comme nous le reverrons plus loin, les patients hystériques n'ont pas le même type de comportement que ceux qui souffrent des mêmes symptômes à la suite d'un réel désordre physiologique. Freud a ainsi mis l'accent sur la différence entre la paralysie biologique et la paralysie hystérique : cette dernière a un caractère très spécifique, d'une extrême intensité, et se limite à une partie du corps. De plus, les comportements moteurs hystériques ne sont pas toujours cohérents. Par exemple, un patient souffrant d'aphonie hystérique n'est plus capable de chuchoter, mais peut tousser, révélant ainsi que ses cordes vocales sont intactes (Spence, 1999, 2001). Quand les patients essaient de bouger, on constate en outre une contraction des muscles agonistes et antagonistes (Merskey, 1995). Enfin, les patients hystériques ne rencontrent pas de difficulté à s'imaginer effectuer un mouvement, contrairement aux patients qui souffrent d'une lésion motrice (Maruff et Velakoulis, 2000). On constate par conséquent une dissociation entre la volonté et l'action automatique, ainsi qu'entre le niveau conscient et le niveau implicite.

On peut aussi soulever la question de savoir si les patients ne feignent pas d'être malades, s'ils ne simulent pas, en raison peut-être du "gain secondaire » qu'ils peuvent en tirer (attirer l'attention sur eux, éviter un travail, etc.). En effet, les simulateurs risquent de présenter le même type d'incohérence motrice que les patients hystériques. Toutefois, la recherche en imagerie cérébrale a permis de montrer des différences physiologiques entre la paralysie feinte et la paralysie hystérique. Spence et coll. (2000) révèlent ainsi une hypoactivité du lobe préfrontal gauche chez les patients hystériques, et du lobe préfrontal droit chez les simulateurs.

L'hystérie ne se confond donc ni avec un désordre physiologique ni avec une maladie feinte. Elle se rapproche par contre de l'hypnose. Le lien entre ces deux phénomènes est double. En premier lieu, Freud et Breuer considéraient l'hypnose comme une méthode permettant de faire ressurgir le trauma psychologique à l'origine de la conversion hystérique. Nous ne nous intéresserons pas ici à cette dimension thérapeutique, mais plutôt aux processus communs mis en action à la fois par l'hypnose et par l'hystérie. Dans les deux cas, il s'agit en effet d'un désordre fonctionnel d'origine psychologique, et non physiologique. Il est possible d'induire sous hypnose le même type de symptômes que l'hystérie : paralysie, douleur, cécité, surdité, etc. Ces symptômes fonctionnels s'accompagnent du même sentiment d'indifférence. De plus, les comportements sous hypnose présentent les mêmes incohérences que dans l'hys- 
térie où les lois normales du comportement sont défiées. Face au conflit entre la réalité et leurs attitudes, les sujets sous hypnose ne sont pas perturbés et cherchent au contraire à le résoudre en ignorant les stimuli externes qui l'ont provoqué. Par exemple, de même que les patients souffrant de cécité hystérique se heurtent rarement aux objets de leur environnement, les sujets sous hypnose disent ne pas voir une poubelle, mais feront un détour pour l'éviter (Bryant et McConkey, 1999). Bryant et McConkey (1995) ont ainsi montré des mots à des sujets aveugles sous hypnose, puis leur ont demandé après la séance de compléter les mots dont seul un fragment était présenté. Les sujets ont de meilleurs résultats si les mots complets leur ont été présentés au préalable alors même qu'ils disaient ne rien voir. Ils conservent par conséquent une connaissance implicite des stimuli visuels qui influencent leur comportement. L'hypnose présente néanmoins une différence majeure par rapport à l'hystérie: les symptômes sont suggérés par autrui et ne durent que peu de temps. Au contraire, l'hystérie présente le paradoxe suivant : le patient n'a pas de contrôle volontaire sur ses symptômes persistants, mais il en est pourtant à l'origine. Oakley (1999) soutient alors que ces deux types de désordre fonctionnel résultent des mêmes processus, même si leur origine est différente, et il propose de qualifier l'hystérie de "désordre par autosuggestion".

L'hystérie est un désordre purement fonctionnel, mais pourtant réel. Cependant, comme pour la schizophrénie, il paraît préférable de se concentrer sur les symptômes plutôt que sur le syndrome lui-même, qui est de plus en plus controversé. C'est pourquoi je m'intéresserai ici principalement à la paralysie hystérique, aux processus en jeu dans celle-ci, mais non à sa cause initiale. En effet, le principe même de conversion reste difficile à comprendre. Comment un trauma psychologique peut-il se transformer en symptôme physiologique ? Pourquoi un patient deviendra-t-il aveugle tandis que l'autre sera paralysé ? L'hystérie met ainsi en cause la nature de la relation entre l'esprit et le corps, mais je n'aborderai pas ce sujet ici. Je me limiterai donc à l'analyse des processus cognitifs sous-jacents à l'hystérie, et plus particulièrement à la paralysie hystérique. À cette fin, je m'appuierai sur les théories neuropsychologiques de certains syndromes se rapprochant de l'hystérie.

\subsection{Les autres troubles de la conscience de nos capacités}

L'hystérie n'est pas sans évoquer deux autres phénomènes pathologiques résultant cette fois-ci d'une lésion cérébrale : la " vision aveugle » [blindsight] et l'anosognosie. Alors que le premier présente le même type de caractéristiques que la cécité hystérique, le second constitue le phénomène miroir de l'hystérie.

À la suite d'une lésion des aires visuelles primaires, les patients souffrant de " vision aveugle » ont l'impression de ne plus voir mais arrivent pourtant à détecter des stimuli, à les localiser, à discriminer des couleurs, des formes et des mouvements dans des situations de choix forcé. Ces capacités résiduelles dépendent d'une voie sous-corticale de l'information visuelle (le système tecto- 
pulvinar). Selon Weiskrantz (1997), l'inconscience des capacités résiduelles s'explique par l'incapacité du patient à soumettre son traitement effectif de l'information visuelle à un " commentaire ", c'est-à-dire à une métareprésentation conceptuelle (Rosenthal, 1993). Sans métareprésentation, le sujet ne peut utiliser de manière volontaire les stimuli visuels traités ou stockés. La vision aveugle peut donc être décrite comme une dissociation entre la conscience et le traitement de l'information visuelle. Il en est de même pour la cécité hystérique. Dans l'étude de Bryant et McConkey (1989), un patient hystériquement aveugle devait déterminer lequel des trois boutons permettait d'arrêter la sonnerie. Sans aucun indice visuel, le patient répondait au hasard. Mais quand le bouton permettant d'arrêter la sonnerie était visuellement différent des autres, il réussissait mieux, et quand il apprit que l'information visuelle influençait son comportement, il réussissait presque parfaitement. Comme la vision aveugle et l'hypnose, la cécité hystérique s'accompagne donc d'un traitement implicite des stimuli visuels. Toutefois, les résultats des patients souffrant de vision aveugle ne s'améliorent pas quand on leur dit s'ils ont répondu correctement ou non contrairement aux résultats des patients hystériques. De plus, la vision aveugle ne s'accompagne pas d'un sentiment d'indifférence vis-à-vis de la maladie, indifférence qui se retrouve au contraire chez les patients anosognosiques.

À la suite d'une lésion cérébrale (le plus souvent au niveau inféropariétal et frontal de l'hémisphère droit), les patients anosognosiques souffrent d'un déficit dont ils n'ont pas conscience. Ce déficit peut être de différents types : visuel (syndrome d'Anton), mnésique, verbal ou moteur. Le terme d'anosognosie a été introduit par Babinski, en 1914, mais le phénomène avait été décrit par Von Monakow dès 1885. Le patient nie sa maladie de manière plus ou moins forte. Il peut reconnaître l'existence d'un problème sans pour autant prendre conscience de son importance et de ses réelles conséquences : par exemple, un patient aveugle refusait d'apprendre le braille, car, selon lui, il n'en avait pas besoin (Sacks, 1996) et il n'associait aucune valeur émotionnelle négative à son déficit (on parle alors d'anosodiaphorie). Il peut aussi le nier et ne pas reconnaître son handicap : les patients paralysés prétendent généralement avoir exécuté le mouvement demandé ou affabulent et expliquent leur immobilité sans faire référence à leur déficit. D'un point de vue externe, le symptôme est le même que dans l'hystérie : les patients sont paralysés dans les deux cas. Mais l'anosognosie se présente comme le phénomène miroir de l'hystérie : dans le premier cas, les patients n'ont pas conscience de leur déficit, tandis que dans le second, ils n'ont pas conscience de leur capacité préservée. Les uns pensent à tort pouvoir se mouvoir, les autres se trompent en se croyant paralysés. Ces deux syndromes illustrent ainsi un trouble de la conscience de la capacité d'agir. Il existe néanmoins une différence entre eux. Alors que les patients hystériques se caractérisent par une dissociation entre le niveau conscient et le niveau implicite, les patients anosognosiques n'ont même pas une connaissance tacite de leur déficit, comme le montre Ramachandran (1995). Il demande à une patiente paralysée d'un seul côté de choisir d'exécuter une tâche qui n'exige 
que l'utilisation de la main intacte, ou une tâche bimanuelle (comme lacer ses chaussures) qui requiert l'utilisation de la main paralysée et de la main intacte. La patiente choisit cette dernière tâche, malgré ses échecs répétitifs. De plus, lorsqu'elle croit voir sa main paralysée bouger (par le biais d'un dispositif expérimental particulier ${ }^{2}$ ), elle ne manifeste aucun signe de surprise.

Nous venons d'étudier deux syndromes neuropsychologiques qui présentent une forte ressemblance avec l'hystérie, même s'ils sont provoqués par une lésion cérébrale et non par un trauma psychologique. D'une part, l'étude de la vision aveugle nous permet de mieux comprendre comment peut subsister un traitement implicite visuel en l'absence de toute conscience phénoménale des stimuli visuels. La dissociation paraît toutefois moins profonde chez les patients hystériques qui sont capables d'améliorer leurs résultats et que leur cécité inquiète peu. D'autre part, l'anosognosie apparaît comme le phénomène inverse de l'hystérie, révélant un trouble de la conscience du déficit. Elle constitue un phénomène largement étudié qui est donc susceptible d'apporter un éclairage nouveau sur l'hystérie et de permettre de mieux comprendre les processus mis en jeu par la conscience de nos propres capacités d'agir.

\section{Agir et pouvoir agir}

Quelle différence existe-t-il entre les rapports introspectifs « j'agis » et « je suis capable d'agir » ou entre " je n'agis pas » et " je suis paralysé " ? Nous pouvons résumer la situation comme suit :

\begin{tabular}{|l|l|l|}
\hline & Conscience de l'acte & Conscience de la capacité \\
\hline Positif & j'agis & $\longrightarrow$ je suis capable d'agir \\
\hline Négatif & je n'agis pas & \\
\hline
\end{tabular}

Ces différentes propositions expriment la conscience de mes propres actions, mais sous des formes différentes. La première colonne porte sur l'occurrence d'un acte ou sur l'absence de cette occurrence, tandis que la seconde décrit une capacité ou un déficit. La conscience de l'acte ne reflète ainsi mon état qu'à un instant donné, alors que la conscience de la capacité d'agir concerne mon état sur une période plus ou moins prolongée. Toutefois, ces deux types de conscience de l'action ne sont pas sans rapport, car l'acte n'est que l'instanciation de la capacité d'agir. Considérons en premier lieu la situation positive. Si j'agis, alors cela signifie que je peux agir et par conséquent que je ne suis pas paralysé. Mais l'inverse n'est pas vrai, car je n'ai pas nécessairement l'intention d'effectuer un mouvement, même si j'en ai la capacité. Au contraire, dans la situation négative, la paralysie implique l'absence de toute action. Mais ce n'est pas réciproque : il est possible de ne pas bouger maintenant pour des raisons

2. Ramachandran utilise une " boîte de réalité virtuelle ", c'est-à-dire une boîte dotée d'un miroir disposé de telle manière que le sujet, en regardant ses mains dans la boîte, voit d'un côté sa main réelle, et de l'autre le reflet de cette main. Le sujet prend donc le reflet de sa main droite pour sa main gauche. Il donne ainsi l'illusion que la patiente peut bouger sa main paralysée. 
autres que la paralysie (par exemple, je n'en ai pas l'intention ou j'en suis momentanément empêché). Par conséquent, la relation entre la conscience de l'acte et la conscience de la capacité d'agir s'inverse dans la situation négative. On peut alors se demander si la conscience de la capacité et la conscience de l'incapacité reposent sur les mêmes processus. Nous approfondirons cette question en l'étudiant plus en détails avec l'anosognosie. Mais je souhaite auparavant insister sur la différence entre agir et pouvoir agir, en la mettant en parallèle avec la distinction entre le schéma corporel et l'image corporelle.

\subsection{Le schéma corporel et l'image corporelle}

L'action se distingue du mouvement au sens où elle est causée par une intention formée par l'agent (Davidson, 1980). Autrement dit, elle est amorcée et guidée tout au long par les représentations motrices du but à atteindre et du mouvement à effectuer. Cette définition néglige toutefois la dimension corporelle. En effet, c'est notre corps qui accomplit nos actions : la représentation du mouvement à effectuer dépend de la position initiale du corps, tandis que nous savons que l'action est réussie si notre corps est dans la position qu'il devait atteindre. En outre, nos actions dépendent de nos capacités corporelles. Une partie du corps peut ne plus obéir à l'agent qui désire se mouvoir (par exemple à la suite d'une lésion de la moelle épinière). Â l'inverse, alors même que le corps est intact, nous pouvons ne plus être capables d'amorcer des mouvements volontaires (comme cela semble être le cas dans l'hystérie). Sans réduire l'une à l'autre, il est néanmoins nécessaire de reconnaître l'interdépendance de la dimension de l'action et de la dimension du corps. Nous pourrions alors proposer que la conscience de l'acte repose en partie sur une représentation dynamique du corps en mouvement, tandis que la conscience de la capacité d'agir dépendrait d'une représentation stable des possibilités corporelles d'agir. Autrement dit, la première dépend d'une image corporelle à court terme, aussi qualifiée de schéma corporel (Head et Holmes, 1911 ; Gallagher, 1995) et la seconde, d'une image corporelle à long terme (O'Shaughnessy, 1995).

Les syndromes neuropsychologiques ont en effet conduit à postuler l'existence de plusieurs types de représentation du corps (Paillard, 1999). Merleau-Ponty (1945) décrit ainsi le cas de Schneider, qui est incapable de décrire la position de son corps ou de localiser les stimulations tactiles, mais qui peut en revanche frotter l'endroit où un moustique l'a piqué. À l'inverse, les patients déafférentés qui ne reçoivent plus aucune information tactile ou proprioceptive sur leur corps restent capables de détecter les sensations de chaud et de froid, de les localiser verbalement et de les situer sur la représentation picturale d'un corps, même s'ils sont incapables de montrer de leur main la partie du corps touchée. Cette double dissociation montre donc l'existence de systèmes distincts de représentation corporelle qui remplissent des fonctions différentes.

3. Ce patient serait probablement considéré de nos jours comme un cas de « toucher insensible " [numbsense], au sens où il peut localiser la piqûre sans en avoir conscience. Pour des cas similaires, voir Rossetti et coll. (1995), et Paillard (1999). 
La vision remplit deux fonctions principales : la reconnaissance [What] et l'action [How]. Cette distinction fonctionnelle se retrouve sur le plan anatomique entre la voie ventrale (inféro-temporale) qui traite les caractéristiques sémantiques de l'objet et la voie dorsale (inféro-pariétale) qui est dédiée aux interactions de l'agent avec l'objet (Milner et Goodale, 1995). Elle peut être aussi appliquée à la représentation du corps. En ce sens, l'image corporelle répond à la question "Quoi ? " (Quelle est cette partie du corps ? Quel est l'état de mon corps ?), tandis que le schéma corporel répond à la question "Comment ? " (Comment utiliser mon corps ? Comment atteindre cette partie du corps ?). Le schéma corporel est utilisé pour agir : il nous permet de marcher sans trébucher ou de prendre un verre, en nous informant sur la position de chacun des segments de notre corps par rapport aux autres et en programmant nos mouvements pour que nous puissions atteindre notre but. Au contraire, l'image corporelle est orientée vers la représentation sémantique et plus détachée du corps. Elle intègre des composantes perceptives, conceptuelles et émotionnelles.

Le schéma corporel est donc une représentation sensori-motrice dynamique qui repose sur un flux d'information continu visuel et somesthésique. En effet, pour agir, il faut connaître à chaque instant l'état et la position des parties du corps de manière très rapide. Le schéma corporel est donc en constante évolution et joue un rôle important dans la conscience de l'acte, en intégrant les informations somatosensorielles nécessaires pour l'action. La conscience que j'agis ne se réduit pas pour autant au seul schéma corporel. En effet, si le médecin lève mon bras, j'ai une représentation de mon bras en mouvement, même si je ne suis pas l'auteur de ce mouvement et si je suis paralysé. Du seul fait que mon bras se lève, je ne peux alors conclure que je suis capable de lever mon bras. Il est alors nécessaire d'intégrer d'autres types d'informations, telles que mon intention d'agir et les anticipations des conséquences sensorielles de mon action. Si mon bras effectue le mouvement que j'avais l'intention d'exécuter et que les résultats de ce mouvement sont ceux que j'avais prédits, alors il est probable que j'en suis à l'origine.

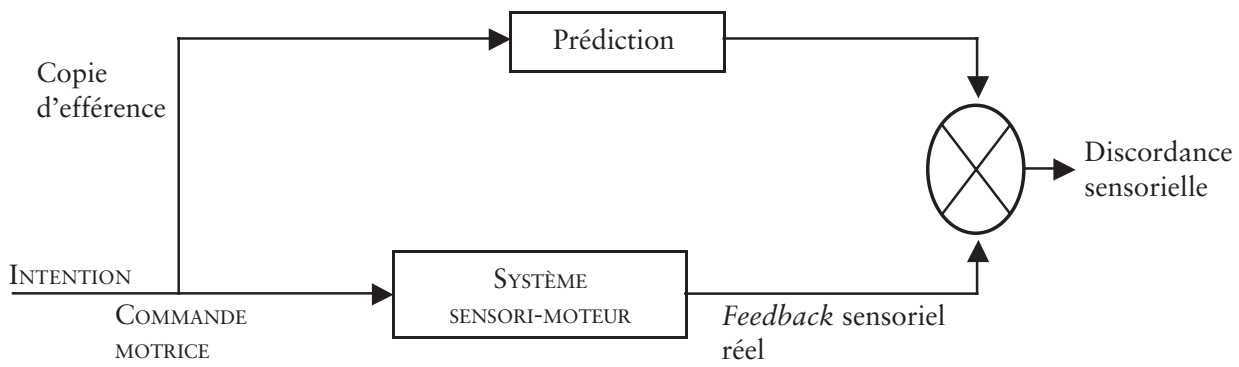

Figure 1. Le modèle de l'action (d'après Blakemore et al., 1999)

La formation d'une intention d'agir conduit à lancer une commande motrice qui acte le système moteur afin de réaliser l'action. Parallèlement, une copie de la commande motrice (ou copie d'efférence) est envoyée pour anticiper les conséquences sensorielles du mouvement. Le contrôle de l'action repose alors sur la comparaison entre les conséquences sensorielles prédites et les réafférences sensorielles effectives. Autrement dit, il s'appuie sur une anticipation et une comparaison de l'état souhaité avec l'état réel. En cas de discordance, le mouvement est rectifié. 
Le schéma corporel ne n'informe toutefois que sur ce que je suis en train de faire maintenant, et non sur ce que je peux faire. La conscience de la capacité d'agir semble alors reposer davantage sur une représentation stable du corps ou, selon la terminologie de Merleau-Ponty (1945), sur le corps habituel plutôt que sur le corps actuel. L'image corporelle pourrait-elle alors jouer ce rôle ? D'un côté, elle constitue une représentation à long terme du corps. Mais de l'autre, elle ne semble pas particulièrement être en jeu dans l'action, puisque c'est là même ce qui la distingue du schéma corporel. Toutefois, il serait faux de croire que l'image corporelle est totalement dissociée du domaine moteur. Ainsi, selon Gallagher et Cole (1995), elle compense l'absence de schéma corporel chez les patients déafférentés pour le contrôle de l'action. Elle jouerait aussi un rôle dans l'apprentissage et le progrès moteur, ainsi que dans l'imitation. Autrement dit, l'image corporelle intervient dans l'action dès que celle-ci demande des réponses conscientes et une perspective réfléchie. Il est alors possible de supposer qu'elle participe à la conscience de la capacité d'agir.

La conscience de la capacité d'agir a ainsi un statut ambigu entre les questions "Quoi ? " et "Comment ? ", statut comparable à celui de la perception visuelle de la fonction d'un objet. En effet, en voyant une fourchette, je peux à la fois la saisir et la manipuler de manière appropriée (je la tiendrai par exemple par le manche plutôt que par les dents) et l'identifier comme étant une fourchette, puisque je connais sa fonction. La fonction d'un objet concerne donc la reconnaissance (quel est cet objet ?) et l'action (comment l'utiliser ?). Il est alors possible de manipuler correctement une fourchette sans savoir à quoi elle sert, comme chez certains patients souffrant d'agnosie visuelle. Inversement, les patients atteints d'ataxie optique ne sont plus capables de l'utiliser correctement, même s'ils en connaissent la fonction. Connaître la fonction d'un objet, c'est alors avoir une information sémantique sur les actions pertinentes que l'on peut réaliser avec cet objet. Autrement dit, c'est avoir une connaissance des dispositions de l'objet. De même, connaître nos propres capacités, c'est avoir une information sémantique sur les actions que notre corps peut exécuter. Il s'agit donc d'une connaissance des dispositions de notre corps, dispositions actualisées dans l'action.

En résumé, la conscience de l'acte et la conscience de la capacité d'agir sont à l'intersection des représentations corporelles et motrices. Le schéma corporel représente alors le corps de manière engagée et pragmatique, tandis que l'image corporelle représente celui-ci de manière détachée et sémantique. Le premier peut être considéré comme un système fonctionnel de capacités motrices mises à exécution (Gallagher, 1995 ; Paillard, 1999), et le second, comme une connaissance sémantique de ces capacités. Il ne faut pas croire pour autant que ces deux types de représentation corporelle fonctionnent de manière purement indépendante. En effet, comme le souligne O'Shaughnessy (1995), elles interagissent continuellement : l'image corporelle structure le schéma corporel, tandis qu'elle est elle-même en partie le résultat de l'extraction d'une représentation du corps commune à tous les schémas corporels. 
Nous allons voir maintenant comment le trouble de l'action et du schéma corporel peut affecter la conscience de la capacité d'agir et l'image corporelle.

\subsection{Le modèle de l'anosognosie}

Nous avons vu que l'anosognosie peut constituer un modèle de compréhension pour l'hystérie. Mais encore faut-il pouvoir expliquer ce syndrome. L'anosognosie soulève en effet trois grandes questions qui pourraient se poser aussi pour l'hystérie : (i) Existe-t-il une influence des facteurs émotionnels et motivationnels ? (ii) Résulte-t-elle d'un déficit spécifique ou d'un problème intellectuel général ? (iii) S'agit-il d'un déficit de haut niveau ou de bas niveau ? (Prigatano et Schacter, 1991). Il est généralement admis de nos jours que l'anosognosie traduit un déficit spécifique et non un phénomène de confusion mentale générale, et qu'il ne s'agit pas d'une défense contre la tension causée par la maladie. De même, Freud considérait que le "gain secondaire " apporté par les symptômes hystériques ne suffit pas pour les justifier et les provoquer. Quel déficit rend alors compte de la perte de la conscience de nos capacités et de nos incapacités ? Comme pour l'hystérie, nous nous intéresserons ici plus spécifiquement à l'anosognosie des déficits moteurs. Une première étape consiste à expliquer comment les patients souffrant d'anosognosie à la suite d'une hémiplégie n'ont pas conscience de ne pas effectuer le mouvement demandé. Il s'agira ensuite de comprendre de manière plus générale comment ils n'ont pas conscience d'être hémiplégiques. Une personne paralysée prend conscience qu'elle ne bouge pas quand elle essaie par exemple de lever son bras et constate que son bras reste immobile. Se produit alors une discordance entre les conséquences sensorielles attendues (le bras levé) et les réafférences sensorielles effectives (le bras immobile). Les patients anosognosiques n'arrivent pas à un tel résultat en raison d'un déficit de ce processus de contrôle de l'action. Comment pourraient-ils alors prendre conscience de leur paralysie, étant donné l'absence de toute discordance ? Le trouble de la conscience de leur incapacité à agir semble alors naturellement découler de la perturbation du suivi de l'action. Expliquer l'anosognosie revient donc à comprendre pourquoi les patients prétendent à tort agir. Comme nous l'avons vu, il faut alors prendre en considération la dimension corporelle et la dimension motrice.

L'anosognosie s'accompagne souvent d'un déficit somatosensoriel. Le patient ne reçoit plus d'information proprioceptive et ne peut donc plus savoir de manière interne que son corps n'est pas en mouvement (même s'il peut le constater visuellement). En outre, l'anosognosie se caractérise dans certains cas - mais non dans tous - par un manque d'attention porté sur une moitié du corps (Bisiach et coll., 1986) ${ }^{4}$. Bisiach propose alors d'expliquer ce syndrome par le dysfonctionnement d'un système de représentation de haut

4. L'héminégligence corporelle se produit à la suite d'une lésion de l'aire pariétale droite et se traduit par exemple par l'oubli de maquiller ou de raser une moitié du visage. 
niveau des performances du sujet, organisé de manière topologique, et spécifique à chaque modalité sensorielle (Bisiach et Berti, 1987). Selon que l'anosognosie s'accompagne ou non d'héminégligence, le système est partiellement détruit (une partie du corps n'étant plus représentée), ou il est confronté à des informations altérées et construit alors une représentation déformée de la réalité. La représentation du côté du corps non lésé peut ainsi parasiter la représentation du côté lésé par un phénomène de complétion : l'ensemble du corps est alors représenté comme intact. Autrement dit, une partie du corps est exclue du schéma corporel ou représentée de manière erronée à partir du modèle de la partie préservée. Bisiach ne distingue pas le schéma corporel et l'image corporelle, mais il semble ici qu'il décrit un déficit du schéma corporel, car c'est la représentation du corps dans son lien avec l'action qui est perturbée.

À cette dimension corporelle, il faut alors ajouter une dimension motrice. En effet, tous les patients paralysés souffrant d'un déficit somatosensoriel ne sont pas pour autant anosognosiques. Selon l'hypothèse "feedforward ", le dysfonctionnement se situe ainsi en amont des réafférences sensorielles, pour ce qui est des intentions d'agir (Heilman, 1991, Adair et coll., 1997, Cole et Paillard, 1995) ${ }^{5}$. Par une forme d'aboulie, le sujet ne veut pas agir et ne s'attend donc pas à certains résultats ; par conséquent il n'y a pas de discordance avec les réafférences sensorielles. Il a été montré en effet qu'un sujet souffrant d'anosognosie ne fait aucun effort pour contracter ses muscles pectoraux quand on lui demande de serrer sa main paralysée, alors qu'il contracte ses muscles des deux côtés quand on lui demande de serrer sa main intacte, ce qui met en évidence une hypoactivité des processus prémoteurs (Gold et al., 1994). L'anosognosie pourrait être ainsi comparée à la négligence motrice qui s'exprime par l'absence de mouvement du côté gauche du corps (Vuilleumier, 2000). L'anosognosie se caractériserait par ce même oubli moteur de la partie paralysée du corps. Nous pourrions alors suggérer que le déficit intentionnel est provoqué par le trouble attentionnel : il m'est impossible de vouloir utiliser la partie de mon corps qui n'est plus représentée dans mon schéma corporel ou du moins à laquelle je ne prête plus attention.

En l'absence de toute discordance entre ses intentions et les réafférences sensorielles, le patient ne peut découvrir qu'il est paralysé, et son image corporelle n'intègre pas le changement quant aux capacités de son corps. Nous pouvons en effet supposer que nous considérons que nous ne sommes pas paralysés à moins d'avoir des preuves suffisantes du contraire. Autrement dit, nous n'aurions pas besoin d'inférer que nous sommes capables de nous mouvoir, il ne s'agirait là que d'une hypothèse par défaut. À l'inverse, nous aurions besoin de "découvrir » que nous sommes paralysés. Ainsi Levine et coll. (1991) proposent que la privation d'information sensorielle n'est pas phénoménologiquement saillante et qu'elle doit être " découverte » par obser-

5. Il faut toutefois remarquer que le modèle de Bisiach refuse l'existence d'un modèle superordonné qui aurait pour fonction de contrôler les défaillances du système, contrairement à l'hypothèse "feedforward". 
vation et par inférences. Or, dans le cas de l'anosognosie pour l'hémiplégie, cette découverte est d'autant plus difficile (i) que la perte est seulement partielle ; (ii) que l'anosognosie s'accompagne souvent d'héminégligence ; et (iii) que le sujet peut faire des inférences incorrectes sur l'état de la partie atteinte à partir de l'état de son côté intact. De même, Ramachandran (1995) soutient que l'hémisphère gauche a pour fonction de résoudre les conflits malgré la présence de petites anomalies localisées de fonctionnement. Or quand l'anomalie est trop importante, une interaction avec l'hémisphère droit provoque un « changement de paradigme » dont le sujet prend conscience. Les patients anosognosiques ne parviendraient alors pas à changer de paradigme, c'està-dire à modifier l'image corporelle qu'ils ont d'eux-mêmes et qui se trouve désormais en décalage par rapport à leur situation réelle.

En résumé, l'anosognosie peut se comprendre en termes d'oubli corporel et moteur. En l'absence d'intention d'agir, le patient n'a pas conscience de ne pas agir, et par conséquent, de ne pas pouvoir agir. Autrement dit, son image corporelle, qui repose en partie sur les souvenirs des actions passées, persiste à représenter les capacités intactes de son corps à se mouvoir. Pourrions-nous proposer une explication similaire pour l'hystérie ? Un " trouble de la volonté » serait donc à l'origine du trouble de la conscience de la capacité d'agir.

\subsection{L'hystérie comme trouble de la volonté}

« Ce ne sont pas les muscles qui refusent d'obéir, mais la volonté elle-même qui a cessé de fonctionner » (Brodie, 1837).

«Ils disent «je ne peux pas»; cela ressemble à «je ne veux pas»; mais c'est «je ne peux pas vouloir»» (Paget, 1873).

L'étude de l'anosognosie nous a montré que le défaut de prise de conscience de l'incapacité d'agir peut s'expliquer par un trouble de l'agir. Il paraît alors intéressant de déterminer si nous pouvons de même expliquer le défaut de prise de conscience de la capacité d'agir par un trouble similaire de l'action. Toutefois, avant d'entrer dans les détails, il faut remarquer que les situations sont légèrement différentes. Comme nous l'avons déjà noté, il semble que par défaut nous pensions que nous ne sommes pas paralysés. Nous n'avons donc pas à activement " prendre conscience " de notre capacité intacte à nous mouvoir, alors qu'il nous faut " découvrir " notre paralysie. Par conséquent, l'hypothèse par défaut semble ne plus être appliquée chez les patients hystériques. Un trouble de l'action similaire à celui de l'anosognosie suffirait-il pour rendre compte de cette particularité ?

Différentes études en imagerie cérébrale ont tenté d'étudier l'hystérie ces dernières années. À cette fin, elles analysent les aires cérébrales spécifiques et les processus cognitifs afférents, lesquels sont mis en jeu quand le patient essaie de mouvoir le membre " paralysé ". Deux modèles principaux s'opposent, mettant l'accent sur un déficit intentionnel ou sur un déficit plus tardif de mise à exécution de l'intention. La première hypothèse cherche à expliquer 
l'hystérie sur le même modèle que l'anosognosie et la négligence motrice. Vuilleumier et coll. (2001) montrent que la paralysie hystérique se caractérise par une hypoactivation des ganglions de la base et du thalamus, qui sont normalement en jeu dans la génération de mouvements volontaires et dans l'apprentissage. Elle se rapproche en cela de la négligence motrice, qui reflète un dysfonctionnement de ces même structures motrices sous-corticales. De plus, des études électrophysiologiques ont montré un patron d'activité cérébrale similaire à celui de la négligence - caractérisé par une modification de l'onde P300 - qui indique un déficit attentionnel (Sierra et Berrios, 2001). De fait, la paralysie hystérique est souvent associée à un déficit somatosensoriel. Il serait ainsi possible de suggérer que la paralysie hystérique est provoquée par un oubli actif d'une partie du corps. Le schéma corporel serait incomplet : le membre n'est plus représenté par rapport aux actions qu'il pourrait accomplir. À ce trouble du schéma corporel s'ajouterait un trouble intentionnel. Comme le dit Paget, les patients seraient incapables de vouloir agir. Spence et coll. (2000) montrent ainsi un dysfonctionnement du cortex préfrontal dorsolatéral gauche (DLPFC), en jeu dans la sélection et la génération de l'action. L'hystérie peut alors se décrire comme une "cessation involontaire des mouvements volontaires » (Spence, 2001) : les patients ne parviennent plus à former l'intention d'agir.

Toutefois, cette hypothèse intentionnelle a été remise en cause par différentes études qui considèrent que le dysfonctionnement s'effectue plus en aval dans la réalisation de l'action. Marshall et coll. (1997) montrent ainsi une activation cérébrale normale quand le patient se prépare à se mouvoir ${ }^{6}$. La différence ne se produit que lorsqu'il essaie de lever sa jambe paralysée : le cortex prémoteur droit ne s'active pas, tandis que l'on constate une hyperactivation du cortex orbito-frontal et du cortex cingulaire antérieur, qui constituent des zones inhibitrices de l'action. De même, Tiihonen et coll. (1995) révèlent une désactivation du cortex sensoriel pariétal associée à une hyperactivation des aires inhibitrices frontales. Selon Athwal et coll. (2001), la paralysie hystérique résulte donc d'un trouble de la mise à exécution de l'intention, et non de l'intention elle-même. Il semble que ce déficit n'apparaisse que lors des mouvements volontaires. En effet, comme nous l'avons vu, la paralysie n'atteint pas certains mouvements automatiques qui sont préservés pour le maintien de l'équilibre ou de la posture. Autrement dit, et ce, de manière paradoxale, c'est la volonté d'agir qui provoquerait la paralysie.

Nous sommes donc confrontés à deux modèles opposés : selon le premier, les patients ne parviennent à former l'intention d'agir ; selon le second, la réalisation de leurs intentions est inhibée. Il est difficile pour un philosophe de trancher, néanmoins je voudrais avancer quelques arguments en faveur de l'hypothèse de l'inhibition. Considérons l'hypothèse du déficit intentionnel.

6. À l'opposé des résultats de Spence et coll. (2000), un fonctionnement normal du cortex préfrontal dorsolatéral a été constaté. 
Pourquoi l'absence d'intention d'agir conduirait-elle à la croyance d'être paralysé ? L'explication de l'anosognosie procédait en deux étapes : l'analyse de la conscience d'agir, puis de la conscience de la capacité (ou de l'incapacité) d'agir. Dans le cas de l'anosognosie, il paraît naturel que le patient ne prenne pas conscience de sa paralysie puisque rien ne remet en cause le fait qu'il croit être capable d'agir. Cependant, dans le cas de l'hystérie, on voit mal comment les mêmes symptômes (l'absence d'intention et le trouble du schéma corporel) pourraient conduire à une conclusion opposée. Le patient hystérique ne constate aucune discordance entre ses intentions et ses mouvements, et, par défaut, il devrait croire qu'il n'est pas paralysé, au même titre que le patient anosognosique.

Il serait alors possible de répondre que l'absence prolongée de mouvement conduit le patient à se sentir paralysé. Mais cet argument ne serait valable que si le patient avait l'intention d'agir, car il n'y a rien de surprenant à ne pas bouger si nous n'en avons pas l'intention. On peut alors remarquer à travers la littérature empirique un certain flou autour des notions de volonté et d'intention d'agir qui sont le plus souvent totalement assimilées. Toutefois, seule l'intention peut véritablement enclencher une action (Davidson, 1980) : le désir est causalement inerte et ne permet pas de comprendre pourquoi l'action est amorcée. De plus, peu d'auteurs reprennent la distinction de Searle (1983) entre les intentions conscientes qui représentent le but général de l'action et qui lui sont antérieures (intentions préalables), et les intentions inconscientes qui représentent les détails du mouvement à exécuter et le guident jusqu'à sa fin (intentions en action). Quelle est l'origine du déficit selon l'hypothèse du déficit intentionnel ? Il semble que le dysfonctionnement de l'intention en action soit en cause, car les patients ont conscience d'avoir l'intention de lever leur jambe ou leur bras. Mais cette hypothèse est alors incompatible avec certains résultats expérimentaux révélant que les patients restent capables d'imaginer qu'ils exécutent des mouvements précis et qu'ils réussissent à le faire aussi bien que les sujets normaux (Maruff et Velakoulis, 2000). Or, selon Searle (1983), l'imagerie motrice reflète la conscience de l'intention en action, qui serait donc intacte chez les patients hystériques. D'autre part, les mouvements automatiques (quand le patient ne prête pas attention à ce qu'il fait) sont préservés. Même si ces mouvements ne sont pas précédés d'une intention préalable consciente, c'est toutefois la présence d'une intention en action qui en assure le suivi. Par conséquent, l'hystérie ne peut se comprendre ni à partir d'un trouble de l'intention préalable, car les patients forment consciemment l'intention d'agir, ni à partir d'un déficit d'intention en action, car ils sont capables non seulement d'imaginer qu'ils se meuvent, mais d'agir de manière automatique. Le dysfonctionnement doit donc se produire plus en aval lors de la mise à exécution de l'intention en action.

Nous pouvons toutefois continuer à qualifier l'hystérie de " trouble de la volonté » si nous l'interprétons non plus comme une forme d'aboulie, mais comme une perte d'efficacité causale de l'intention. En effet, il ne suffit pas 
que l'action corresponde au contenu de l'intention, il faut que l'intention soit la cause de l'action. Selon Searle, l'intention spécifie qu'elle est la cause de l'action, et elle fait référence à elle-même de manière causale [causal self-referentiality]. D'une part, l'intention préalable est la cause de l'action. D'autre part, l'intention en action est une composante de l'action et elle est la cause du mouvement corporel grâce auquel l'action est accomplie. Dans l'hystérie, il semble que l'efficacité causale de l'intention en action soit inhibée : l'intention ne parvient plus à engendrer le mouvement corporel. Plus précisément, elle n'est inhibée que pour les mouvements volontaires. De manière très spéculative, on pourrait alors imaginer que la formation d'une intention préalable provoque non seulement la formation d'une intention en action, mais aussi de manière paradoxale, une inhibition de l'efficacité causale de cette dernière.

Nous venons d'examiner les processus cognitifs possibles sous-jacents à la paralysie hystérique. Il faut maintenant nous intéresser à la seconde étape de notre analyse, à savoir les raisons pour lesquelles les patients se croient paralysés. Selon une première interprétation, que nous qualifierons d' " ascendante » [bottom-up], le dysfonctionnement de l'action explique la croyance en la paralysie. Autrement dit, les patients forment certaines intentions d'agir, anticipent les conséquences sensorielles de leurs actions et constatent qu'elles ne correspondent pas à la réalité, car l'exécution des mouvements a été inhibée. Confrontés à cette discordance, ils concluent qu'ils ne sont plus capables de se mouvoir et modifient en conséquence leur image corporelle. Mais il est possible d'offrir une autre interprétation de plus haut niveau. Nous avons jusqu'à présent constaté la présence d'une inhibition de l'action, mais nous n'avons pas cherché à en comprendre l'origine. Or nous pourrions imaginer qu'elle est due à l'image corporelle que les patients ont d'euxmêmes. Nous avons vu que l'absence de représentation d'une partie du corps dans le schéma corporel pouvait conduire à une forme de négligence motrice, voire d'anosognosie. De même, il est possible qu'un trouble de l'image corporelle induise une certaine paralysie hystérique. En effet, un trauma psychologique peut influencer la composante émotionnelle de l'image corporelle. D'autres syndromes psychopathologiques, par exemple l'anorexie mentale, trouvent de même leur origine dans une perturbation de l'image corporelle. Selon cette interprétation " descendante " [top-down], les patients se perçoivent comme étant paralysés, et le deviennent par conséquent, en inhibant tout mouvement volontaire.

L'hystérie constitue alors le phénomène miroir de l'anosognosie. En effet, alors que la première se comprend à partir d'une modification pathologique de l'image corporelle qui conduit le patient à se croire paralysé, la seconde s'explique par une préservation de l'image corporelle telle qu'elle était avant la paralysie, conduisant le patient à croire qu'il n'a pas changé. Autrement dit, dans le premier cas, l'image corporelle change alors qu'elle ne le devrait pas, alors que dans le second, elle ne change pas alors qu'elle le devrait. 
La réalité de l'hystérie de conversion a été l'objet d'un long débat entre ceux qui affirment qu'une telle entité clinique n'est qu'un produit culturel ou n'existe que dans l'œil du médecin, et ceux qui persistent à en reconnaître l'existence, ou du moins, à analyser certains de ses symptômes tels que la paralysie hystérique. Au même titre que l'anosognosie, l'hypnose ou la vision aveugle, cette pathologie révèle la possibilité d'une dissociation entre notre conscience et nos capacités, et permet d'approfondir la notion de conscience de soi comme agent et comme corps en mouvement. La paralysie hystérique se comprend alors à partir d'un trouble de la conscience de la capacité d'agir et de l'image corporelle, dans leurs relations avec la conscience d'agir et le schéma corporel. Elle constitue le phénomène miroir de l'anosognosie, syndrome où le patient conclut qu'il n'est pas paralysé du fait qu'il pense se mouvoir (ou du moins du fait que rien n'indique le contraire). Son image corporelle ne reflète alors pas l'altération de son schéma corporel. Au contraire, dans l'hystérie, la croyance en la paralysie qui découle de la perturbation de l'image corporelle a pour conséquence une incapacité réelle du patient à agir. Certaines questions sur la nature de l'hystérie restent néanmoins ouvertes, et nous sommes encore loin d'en établir les conditions nécessaires et suffisantes.

\section{Bibliographie}

Adair, J.C., R.L. Schwartz, D.L. Na, E. Fennell, R.L. Gilmore, et K.M. Heilman. "Anosognosia : Examining the Disconnection Hypothesis », J Neurol Neurosurg Psychiatry, 63, 1997, p. 798-800.

Athwal, B.S., P.W. Halligan, G.R. Fink, J.C. Marshall, et R.S.J. Frackowiak. « Imaging Hysterical Paralysis ", dans P.W. Halligan, C. Bass et A.S. David, dir., Contemporary Approaches to the Study of Hysteria, New York, Oxford University Press, 2001, p. 216-234.

Bisiach, E., G. Vallar, D. Perani, C. Papagno, et A. Berti. « Unawareness of Disease Following Lesions of the Right Hemisphere : Anosognosia for Hemiplegia and Anosognosia for Hemianopia ", Neuropsychologia, 24(4), 1986, p. 471-482.

Bisiach, E. et A. Berti. "Dyschiria. An Attempt at its Systemic Explanation ", dans M. Jeannerod, dir., Neurophysiological and neuropsychological aspects of spatial neglect, Amsterdam, Elsevier, 1987.

Blakemore, S., C. Frith, et D. Wolpert, "Spatiotemporal Prediction Modulates the Perception of Self Produced Stimuli », Journal of Cognitive Neuroscience, 11, 1999, p. 551-559.

Brodie, B.C. Lectures Illustrative of Certain Nervous Affections, Londres, Longman, 1837.

Bryant, R.A. et K.M. McConkey. « Visual Conversion Disorder : a Case Analysis of the Influence of Visual Information ", Journal of Abnormal Psychology, 98, 1989, p. 326-329.

Bryant R.A. et K.M. McConkey. « Hypnotic Blindness and the Priming Effect of Visual Information ", Contemporary Hypnosis, 12, 1995, p. 157-164.

- . "Functional Blindness : a Construction of Cognitive and Social Influences ", dans P.W. Halligan et A.S. David, dir., Conversion Hysteria : Towards a Cognitive Neuropsychological Account, Cognitive Neuropsychiatry, Hove, Psychology Press, 1999, p. 227-242. 
Carter, R.B. On the Pathology and Treatment of Hysteria, Londres, Churchill, 1853.

Charcot, J.M. Clinical Lectures on the Diseases of the Nervous System, vol III, Londres, New Sydenham Society, 1889.

Cole, J. et J. Paillard. « Living Without Touch and Peripheral Information About Body Position and Movement : Studies with Deafferented Subjects ", dans J.L. Bermudez, A. Marcel et N. Eilan, dir., the Body and the Self, Cambridge, Mass., MIT Press, 1995.

Davidson, D. Essays on Actions and Events, Oxford, Oxford University Press, 1980. Ferriar, J. Medical Histories and Reflections, Vol II, Londres, Cadell and Davies, 1795.

Freud, S. "The Neuropsychoses of Defence ", dans The Complete Psychological Works, vol III, Londres, Hogarth, 1894, p. 45-61.

Frith, C.D. The Cognitive Neuropsychology of Schizophrenia, Hove, Lawrence Erlbaum Associates, 1992.

Gallagher, S. " Body Schema and Intentionality », dans J.L. Bermudez, A. Marcel et N. Eilan, dir., The Body and the Self, Cambridge, Mass., MIT Press, 1995.

Gallagher, S. et J. Cole. " Body Schema and Body Image in a Deafferented Subject », Journal of Mind and Behavior, 16 (4), 1995, p. 369-390.

Gold, M., J.C. Adair, D.H. Jacobs, et K.M. Heilman. " Anosognosia for Hemiplegia : an Electrophysiologic Investigation of the Feed-Forward Hypothesis », Neurology, 44 (10), 1994, p. 1804-1808.

Graham, G. et G.L. Stephens. dir., Philosophical Psychopathology, Cambridge, Mass., MIT Press, 1994.

Head, H. et G. Holmes. « Sensory Disturbances from Cerebral Lesions », Brain, 34, 1911, p. 102-254.

Heilman, K.M. "Anosognosia : Possible Neuropsychological Mechanisms », dans G.P. Prigatano et D.L. Schacter, dir., Awareness of Deficit After Brain Injury. Clinical and Theoretical Issues, New York, Oxford University Press, 1991.

Janet, P. The Majors Symptoms of Hysteria, New York, MacMillan, 1907.

Levine, D.N., R. Calvanio, et W.E. Rinn. "The Pathogenesis of Anosognosia for Hemiplegia », Neurology, 41 (11), 1991, p. 1770-1781.

Mace, C. "All in the Mind ? the History of Hysterical Conversion as a Clinical Concept ", dans P.W. Halligan, C. Bass et A.S. David, dir., Contemporary Approaches to the Study of Hysteria, New York, Oxford University Press, 2001, p. 1-11.

Marsden, C.D. "Hysteria - A Neurologist's View », Psychological Medicine, 16(2), 1986, p. 277-288.

Marshall, J.C., P.W. Halligan, G.R. Fink, D.T. Wade, et R.S.J. Frackowiak. «The Functional Anatomy of Hysterical Paralysis », Cognition, 64, 1997, p. B1-B8.

Maruff, P. et D. Velakoulis. "The Voluntary Control of Motor Imagery. Imagined Movements in Individuals with Feigned Motor Impairment and Conversion Disorder ", Neuropsychologia, 38, 2000, p. 1251-1260.

Merleau-Ponty, M. Phénoménologie de la perception, Paris, Gallimard, 1945.

Merskey, H. "The Analysis of Hysteria : Understanding Conversion and Dissociation ", Londres, 1995.

Miller, E. "Conversion Hysteria : is it a Viable Concept ? ", dans P.W. Halligan et A.S. David, dir., Conversion Hysteria : Towards a Cognitive Neuropsychological Account, Cognitive Neuropsychiatry, Hove, Psychology Press, 1999, p. 181-192.

Milner, D. et M.A. Goodale. "The Visual Brain In Action », New York, Oxford University Press, 1995. 
Oakley, D.A. "Hypnosis and Conversion Hysteria : a Unifying Model », dans P.W. Halligan et A.S. David, dir., Conversion Hysteria : Towards a Cognitive Neuropsychological Account, Cognitive Neuropsychiatry, Hove, Psychology Press, 1999, p. 243-266.

O'Shaughnessy, B. "Proprioception and the Body Image ", dans J.L. Bermudez, A. Marcel et N. Eilan, dir., The Body and the Self, Cambridge, Mass., MIT Press, 1995.

Paillard, J. « Body Schema and Body Image - A Double Dissociation in Deafferented Patients ", dans G.N. Gantchev, S. Mori et J. Massion, Sophia, dir., Motor Control, Today and Tomorrow, Academic Publishing House, 1999.

Prigatano, G.P. et D.L. Schacter. «Introduction », dans G.P. Prigatano et D.L. Schacter, dir., Awareness of Deficit After Brain Injury. Clinical and Theoretical Issues, New York, Oxford University Press, 1991.

Proust, J. «Awareness of Agency : Three Levels of Analysis », dans T. Metzinger, dir., The Neural Correlates of Consciousness, Cambridge, Mass., MIT Press, 2000.

Ramachandran, V.S. "Anosognosia in Parietal Lobe Syndrome ", Consciousness and Cognition, 4, 1995, p. 22-51.

Rosenthal, D.M. " Thinking that One Thinks », dans M. Davies et G.W. Humphrey, dir., Consciousness, Oxford, Blackwell, 1993, p. 197-223.

Rossetti, Y., G. Rode, et D. Boisson. "Implicit Processing of Somaesthetic Information : a Dissociation Between Where and How ? ", Neuroreport, 6(3), 1995, p. 506-10.

Sacks, O. Un anthropologue sur Mars, Paris, Seuil, 1996.

Searle, J. Intentionality, Cambridge, Cambridge University Press, 1983.

Sierra, M. et G.E. Berrios. "Conversion Hysteria : the Relevance of Attentional Awareness », dans P.W. Halligan, C. Bass et A.S. David, dir., Contemporary Approaches to the Study of Hysteria, New York, Oxford University Press, 2001, p. 192-202.

Slater, E. et E. Glithero. "A Follow-Up of Patients Diagnosed as Suffering from 'Hysteria' ", Journal of Psychosomatic Research, 9, 1965, p. 9-11.

Spence, S.A. "Hysterical Paralyses as Disorders of Action ", dans P.W. Halligan et A.S. David, dir., Conversion Hysteria : Towards a Cognitive Neuropsychological Account, Cognitive Neuropsychiatry, Hove, Psychology Press, 1999, p. 203-226.

Spence, S.A. " Disorders of Willed Action ", dans P.W. Halligan, C. Bass et A.S. David, dir., Contemporary Approaches to the Study of Hysteria, New York, Oxford University Press, 2001, p. 235-250.

Spence, S.A., H.L. Crimlisk, H. Cope, M.R. Ron, et P.M. Grasby. « Discrete Neurophysiological Correlates in Prefrontal Cortex During Hysterical and Feigned Disorder of Movement ", The Lancet, 355, 2000, p. 1243-1244.

Tiihonen, J., J. Kuikka, H. Viinamaki et al. « Altered Cerebral Blood Flow During Hysterical Paraesthesia ", Biological Psychiatry, 37, 1995, p. 134-135.

de Vignemont, F. et P. Fourneret. "The Sense of Agency : a Philosophical and Empirical Review of the 'Who' System », Consciousness and Cognition, 2003.

Vuilleumier, P. "Anosognosia ", dans J. Bogousslavsky et J.L. Cummings, dir., Behavior and Mood Disorders in Focal Brain Lesions, Cambridge, Cambridge University Press, 2000, p. 465-519.

Vuilleumier, P., C. Chicherio, F. Assal, S. Schwartz, D. Slosman, et T. Landis. "Functional Neuronanatomical Correlates of Hysterical Sensorimotor Loss ", Brain, 124, 2001, p. 1077-1090.

Weiskrantz, L. The Conscience Lost and Found, New York, Oxford University Press, 1997. 